\section{Manual versus automated image fusion of real-time ultrasonography and MR/CT images for radiofrequency ablation of hepatic tumors: results of a randomized prospective trial (NCT02705118)}

\author{
Moon Hyung Choi ${ }^{1,2}$, Joon-II Choi ${ }^{2,3}$, Young Joon Lee ${ }^{1}$ \\ 'Department of Radiology, Eunpyeong St. Mary's Hospital, College of Medicine, The Catholic \\ University of Korea, Seoul; ${ }^{2}$ Cancer Research Institute of the Catholic University of Korea, \\ Seoul; ${ }^{3}$ Department of Radiology, Seoul St. Mary's Hospital, College of Medicine, The Catholic \\ University of Korea, Seoul, Korea
}

Purpose: This study compared the technical parameters and clinical outcomes of manual and automatic image fusion techniques of ultrasonography and magnetic resonance imaging/ computed tomography for radiofrequency ablation (RFA) of hepatic tumors.

Methods: Seventy consecutive patients (male:female $=47: 23,67.1 \pm 10.9$ years old) who underwent RFA for hepatic tumors were prospectively enrolled and randomly assigned to the manual or automatic registration group. Two operators performed RFA with one of two imaging fusion techniques. Technical parameters (the registration error, time required for image registration, number of point registrations) and clinical outcomes (technical success, technical effectiveness, local tumor progression [LTP]-free survival, and progression-free survival [PFS]) were compared.

Results: The automatic group contained 35 patients with hepatocellular carcinoma, while the manual group included 34 hepatocellular carcinoma patients and a patient with colon cancer liver metastasis. The registration error, time required for registration, and number of point registrations were $5.7 \pm 4.3 \mathrm{~mm}, 147.8 \pm 78.2$ seconds, and $3.26 \pm 1.20$ in the automatic group, and $6.3 \pm 5.0 \mathrm{~mm}, 150.3 \pm 89.7$ seconds, and $3.20 \pm 1.13$ in the manual group, respectively. The technical success and effectiveness rates were both $97.1 \%$ in the automatic group and both $100.0 \%$ in the manual group. The above differences were not significant. The LTP-free survival and PFS (28.3 and 21.2 months in the automatic group, and 29.0 and 24.9 months in the manual group, respectively) showed no significant between-group differences during a median 20.1-month follow-up period.

Conclusion: The technical parameters and clinical outcomes of automatic image fusion were not significantly different from those of manual image fusion for RFA of hepatic tumors.

Keywords: Liver; Radiofrequency ablation; Image fusion; Ultrasonography

e-ultrasonography.org

Ultrasonography 40(2), April 2021

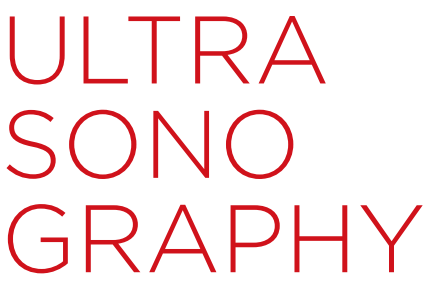

ORIGINAL ARTICLE

https://doi.org/10.14366/usg.20052 pISSN: 2288-5919 - eISSN: 2288-5943

Ultrasonography 2021;40:237-247

Received: April 8, 2020

Revised: May 19, 2020

Accepted: May 21, 2020

Correspondence to:

Joon-II Choi, MD, Department of Radiology, Seoul St. Mary's Hospital, College of Medicine, The Catholic University of Korea, 222 Banpo-daero, Seocho-gu, Seoul 06591, Korea

Tel. +82-2-2258-1431

Fax. +82-2-599-6771

E-mail: dumkycji@gmail.com

This is an Open Access article distributed under the terms of the Creative Commons Attribution NonCommercial License (http://creativecommons.org/ licenses/by-nc/4.0/) which permits unrestricted noncommercial use, distribution, and reproduction in any medium, provided the original work is properly cited.

Copyright (? 2021 Korean Society of Ultrasound in Medicine (KSUM)

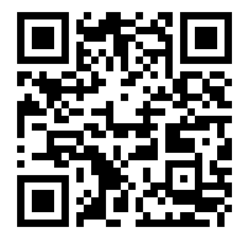

How to cite this article:

Choi MH, Choi Jl, Lee YJ. Manual versus automated image fusion of real-time ultrasonography and MR/CT images for radiofrequency ablation of hepatic tumors: results of a randomized prospective trial (NCT02705118). Ultrasonography. 2021 Apr;40(2):237-247. 


\section{Introduction}

Radiofrequency ablation (RFA) is now widely accepted as an effective locoregional treatment modality for small hepatocellular carcinomas (HCC) and colorectal cancer liver metastasis (CRLM) by most major scientific bodies [1-4]. Ultrasonography (US) is the most commonly used guiding modality for percutaneous RFA, especially in Asian countries, due to its advantages such as convenience, ready availability, lack of radiation hazard, lower cost, and capability for real-time control $[5,6]$. However, US guidance for small hepatic tumors is difficult in some situations, including cases where there is a limited sonic window, too many hepatic nodules in cirrhotic liver parenchyma or isoechogenicity of the tumor to the background liver, and invisibility of $30 \%-55 \%$ of the target lesions on US $[5,7-10]$. Such situations are more frequently encountered in patients with advanced liver cirrhosis, who are at an elevated risk of developing HCC $[7,10,11]$.

Several fusion imaging techniques, which can combine real-time US images with computed tomography (CT) or magnetic resonance imaging (MRI) findings acquired in advance, have been developed by many US vendors to overcome this shortcoming of US guidance [12-17]. Fusion imaging can enhance the visibility of small tumors on US while preserving the advantages of US guidance, including real-time guidance and monitoring. However, less-experienced operators do not find it easy to perform fusion imaging techniques in daily practice. The majority of the fusion techniques require manual registration, which consists of plane and point registration. Plane registration is time-consuming because finding the same planes on US and CT/MRI is sometimes difficult for less-experienced operators.

The recently developed automatic registration technique can simplify the process of plane registration [18-24], because this technique can automatically register the same planes on US and CT/MRI. Cha et al. [19] reported that the registration errors of manual and automatic registration techniques were similar and the time required for image fusion was significantly shorter with the automatic registration technique. Thus, based on previous research, it is possible that automatic registration could reduce registration time without losing registration accuracy. Conversely, for radiologists who are familiar with manual registration, automatic registration may not be very beneficial. To our knowledge, no study has yet compared the technical parameters and clinical outcomes of the automatic and manual registration techniques for RFA of hepatic tumors between radiologists with different levels of experience. We hypothesized that automatic registration might ease US-CT/MRI image fusion procedures and improve the clinical outcomes of RFA of hepatic tumors, especially for less-experienced radiologists.
Therefore, the purpose of this trial was to compare the technical parameters, including the registration time and accuracy, and the clinical outcomes of manual and automatic image fusion systems for RFA of hepatic tumors.

\section{Materials and Methods}

\section{Inclusion Criteria, Sample Size Calculation, and Randomization}

This study was a single-center, prospective randomized trial. The institutional review board of our institution approved the study protocol and written consent was obtained from all patients. This trial was registered at ClinicalTrials.gov (register number: NCT02705118).

Patients older than 40 years of age who planned to undergo RFA of hepatic tumors after February 2016 were eligible to participate. The inclusion criteria were as follows: (1) HCC or CRLM, (2) tumors $\leq 4 \mathrm{~cm}$ and less than 3 in number, and (3) RFA performed under US guidance with image fusion. The exclusion criteria were as follows: (1) RFA performed under CT or fluoroscopic guidance, (2) cholangiocarcinoma or hepatic metastasis not from colorectal cancer, or (3) refusal to participate in this study. The diagnosis of HCC was made by histology or based on the typical imaging findings of HCC defined by the American Association for the Study of the Liver Disease or European Association for the Study of the Liver $[25,26]$. We also adopted the guideline for diagnosing HCC published by the Korean Liver Cancer Association and National Cancer Center Korea, because gadoxetic acid-enhanced MRI was performed for most patients at our institution [27]. The diagnosis of CRLM was made based on a pathological examination.

To calculate the sample size, we hypothesized that the difference of time required for registration between manual and automatic registration would be at least 20 seconds. This is because plane registration for the automatic registration takes roughly 5 seconds, while that of manual registration usually needs more than 25 seconds. We also hypothesized that the standard deviation of the registration time would be approximately 25 seconds based on a previously published study by Lee et al. [28]. A sample size of 34 patients for each group was calculated to obtain $90 \%$ statistical power with statistical significance of 0.05 . Assuming a 20\% dropout rate, a total sample size of 82 patients was required. The sample size was calculated using MedCalc version 17.7 (MedCalc Software, Mariakerke, Belgium).

The patients were randomly assigned to undergo either manual registration or automatic registration in a 1:1 ratio after signing a written informed consent form. Block randomization was performed and the assignment number was generated by an independent statistician to maximize the comparability and to rule out the 
influence of the researchers' subjective perceptions. Randomization was performed using SAS for Windows version 9.3 (SAS Institute Inc, Cary, NC, USA).

\section{Acquisition of MRI and CT}

For image fusion, we used MRI or CT scans obtained within 1 month of RFA to avoid changes in target lesions. If both CT and MRI data were available, we preferred MRI because the index tumors and/or surrounding vessels, which can be landmarks for image registration, are very conspicuous on hepatobiliary-phase images of gadoxetic acid-enhanced MRI [29]. When MRI was not available, we used latearterial-phase $\mathrm{CT}$ images for image fusion.

All MRI scans were performed using a 3-T MRI scanner (Magnetom Verio, Siemens Healthineers, Erlangen, Germany) at our institution with an 8-channel phased array coil. The included sequences were (1) T2-weighted images using fast spin echo with fat suppression; (2) T2-weighted images using single-shot fast spin echo with fat suppression; (3) T1-weighted images with in- and opposed-phase dual gradient echo; (4) diffusion-weighted images using echo-planar imaging, applying b-values of $0,50,500$, and $800 \mathrm{~s} / \mathrm{mm}^{2}$; and (5) dynamic imaging using three-dimensional gradient-recalled echo imaging. Dynamic imaging consists of non-contrast, late arterial (30-35 seconds delay with the bolus-tracking technique), portal venous (65-80 seconds delay), transitional (180 seconds delay) and hepatobiliary phases (20 minutes delay) using gadoxetic acid.

The CT scans were acquired with a 128- or 64-slice CT scanner (Somatom Definition/Somatom Definition AS+, Siemens Healthineers; Discovery CT750 HD, GE Healthcare, Chicago, IL, USA) using a slice thickness of $5 \mathrm{~mm}$ with a 5 -mm interval for all phases.

\section{Operators and US Systems for Image Fusion}

A senior interventional radiologist with 13 years of experience in RFA ( $>1,000$ cases of RFA, $>200$ cases of imaging fusion) and a junior interventional radiologist, who was less experienced, with 3 years of experience in RFA ( $>50$ cases of RFA, $>30$ cases of imaging fusion), participated in this study. They performed more than 10 cases of RFA or biopsy with automatic registration for 3 months before enrolling patients to get used to the new automatic registration technique.

The RS80A US system (Samsung Medison, Seoul, Korea), which is capable of fusion imaging (S-Fusion, Samsung Medison) was adopted for automatic registration. The LOGIQ E9 US system (GE Healthcare), which is also capable of fusion imaging (Volume Navigation, GE Healthcare) was adopted for manual registration. These US systems were coupled with a magnetic field generator. Two electromagnetic position sensors were connected to a position sensing unit and were attached on a convex-type ultrasound transducer using a bracket. Both the transmitter and sensors were connected to a fully integrated position sensor unit embedded in the US unit (Ascension Technology, Shelburne, VT, USA). For the LOGIQ E9 US system, an active patient tracker (OmniTrax, CIVCO Medical Solution, Kalona, IA, USA) was attached on the left anterior chest wall of the patient to correct patient motion.

\section{Manual Registration}

To perform fusion imaging of real-time US and CT/MRI, Digital Imaging and Communications in Medicine data from CT/MRI should be uploaded to the US unit. Manual registration is performed after uploading the imaging data. Manual registration consists of the following two steps: plane registration and point registration. Plane registration is a process of finding the same planes on US and CT/MRI to enable the US system to identify the position and direction of the US transducer regarding the patient's body. For plane registration, the coronal plane is usually used when an index tumor is located at the right hemiliver. The axial plane can be used when the index tumor is located in the left hemiliver. After plane registration, point registration is performed by identifying the same points on both imaging modalities (US and CT/MRI) for finetuning of the registration process. Anatomical structures such as bifurcation of vessels or cysts are very useful for point registration. Point registration can be performed as extensively as needed until the operator is satisfied with the result of the registration. We did not use an index tumor as a landmark for the point registration because we wanted to measure the registration error. The operators also recorded the presence of cirrhosis, the quality of the sonic window (good or poor), and the visibility of index tumors on grayscale US images using a 4-point scale (very good, good, moderate, and poor). The visibility of the index tumor was determined based on the degree to which the tumor boundary was well distinguished before image fusion. Tumor visibility was included in the analysis of patients' characteristics to make sure that the distribution of clearly visible tumors was not different between the automatic and manual registration groups.

\section{Automatic Registration}

We used the positioning auto-registration function of the RS80A US system for automatic registration [18-21]. The positioning auto-registration function also consists of plane registration and point registration, similar to manual registration. However, plane registration can be done very quickly and conveniently with this technique. For plane registration in the automatic registration technique, the operator simply positions the US transducer on top of the solar plexus (the junction between the body of the sternum and xiphoid process) in the sagittal plane and presses the "registration" 
button. By doing this, the US system is informed of the location (on the solar plexus) and direction (sagittal plane) of the transducer and performs the plane registration automatically. After plane registration, the operator performs the first point registration at the inferior tip of the left hemiliver on the midline sagittal plane. The inferior tip of the liver can easily be found on the sagittal plane immediately after plane registration (Fig. 1). The operator can then perform the point registration as much as he or she needs to optimize the registration of the images. Likewise, the operators recorded the presence of cirrhosis, the quality of the sonic window (good or poor), and the visibility of index tumors on gray-scale US images with a 4-point scale.

\section{Endpoints}

The primary endpoints were the technical parameters of the registration, including registration accuracy (measured by registration error), registration time, and the number of point registrations. The secondary endpoints were clinical outcomes including the technical success rate, the technical effectiveness rate 1 month after RFA, local tumor progression (LTP)-free survival, and progression-free survival (PFS).

To assess the registration error, we measured the distance between the center of an index tumor on both imaging modality (US and (T/MRI) after completion of image registration (Fig. 2). Patients were asked to hold their breath to minimize the registration error. The operators measured the registration error in the largest index tumor per patient because the registration of the second or third index tumors required additional point registrations.

We measured the required time for registration using the timer of the US system; specifically, it was calculated from the time when the upload of the images to the US system had been completed to the completion of image registration. We also recorded the number of

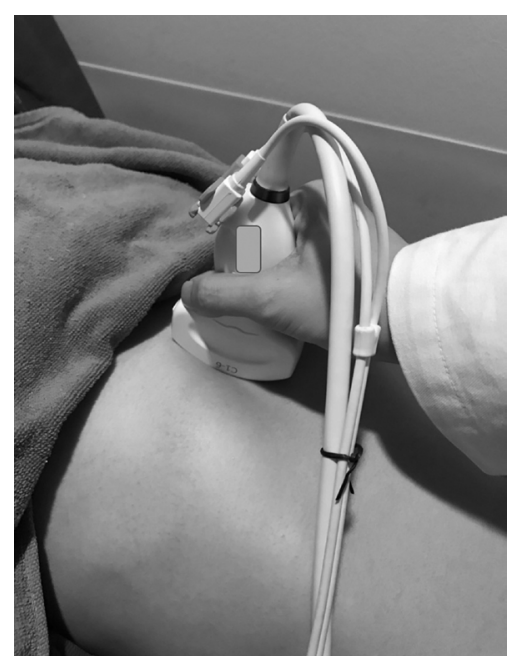

A

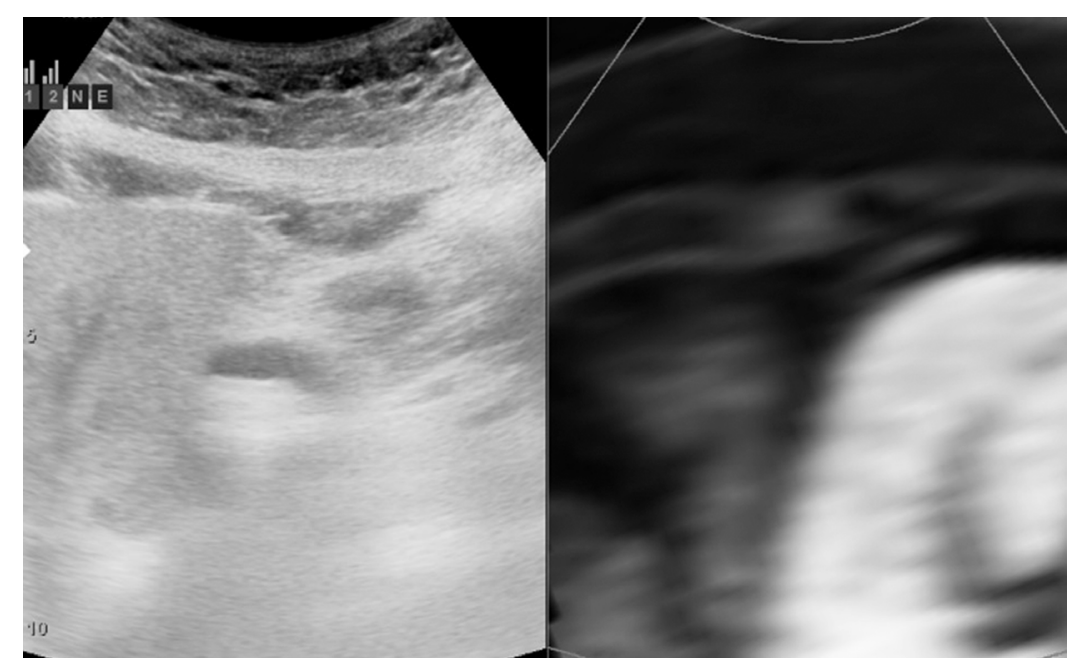

B

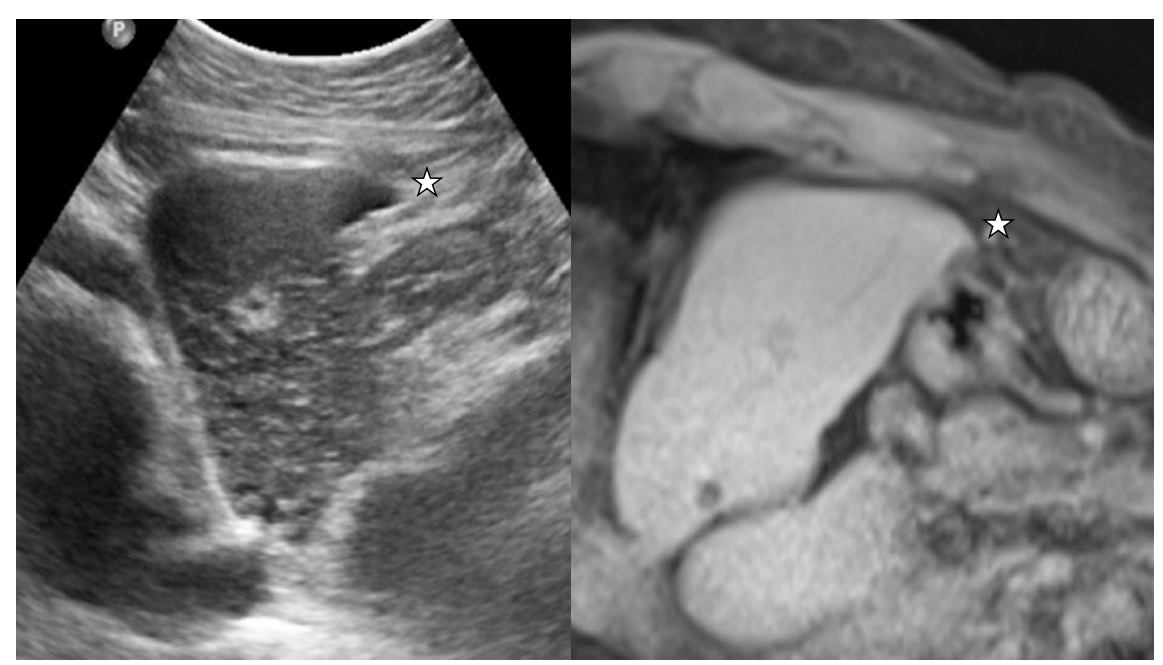

C
Fig. 1. The method of automatic registration. A. A convex transducer with an electromagnetic sensor was placed on top of the solar plexus on the sagittal plane. The "registration" button was then pressed. B. On the sagittal plane, the left lateral sector of the liver was visualized on ultrasonography and fused with magnetic resonance imaging. C. First, point registration was done at the inferior tip of the left liver (star). Then, multiple additional point registrations could be done for fine-tuning of the registration. 
point registrations for the index tumor in each patient.

To evaluate technical success, all patients underwent CT within 24 hours after RFA. The CT protocols were as the same as those of the pre-RFA CT scans. The radiologist who performed RFA reviewed the CT scans of the same patient to evaluate the presence of a residual tumor or the occurrence of immediate complications. Technical success was defined as complete ablation of the index tumor and the absence of a viable residual tumor. Technical effectiveness was evaluated using follow-up CT scans 1 month after RFA, and was defined in the same way as technical success. The patients then usually underwent follow-up CT scans at 3-month intervals to evaluate the presence of LTP or recurrence distant to the treated target lesions.

\section{Statistical Analyses}

Patient characteristics were compared between the two groups using the Student t-test for continuous variables and the Fisher exact test for categorical variables. The visibility of the index tumors on gray-scale imaging was analyzed using the chi-square test. The registration error, time for image registration and the number of point registrations for both methods were compared using the Student t-test for the overall analyses and the Mann Whitney $\mathrm{U}$ test for subgroup analyses. The technical success rate and the technical effectiveness rate in both groups were compared using the Fisher exact test. For survival analysis, we used the Kaplan-Meier method with the log-rank test for LTP-free survival and PFS. Two-sided $\mathrm{P}$-values of less than 0.05 were considered to indicate statistically

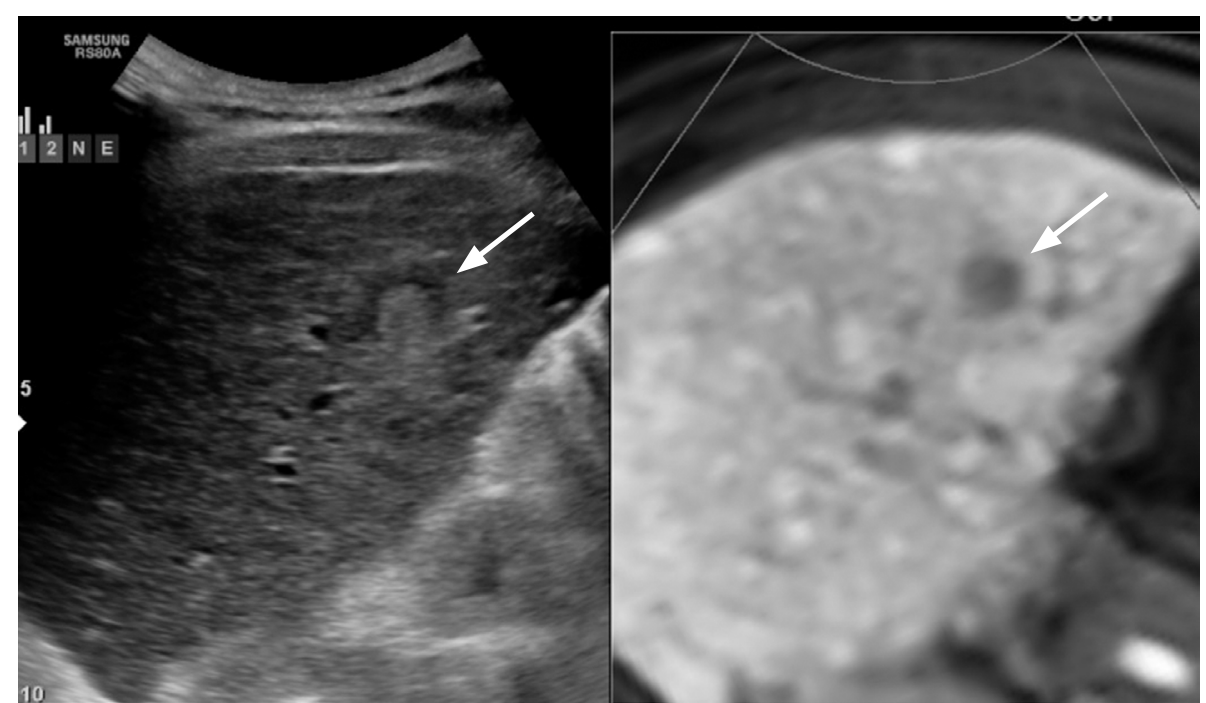

A

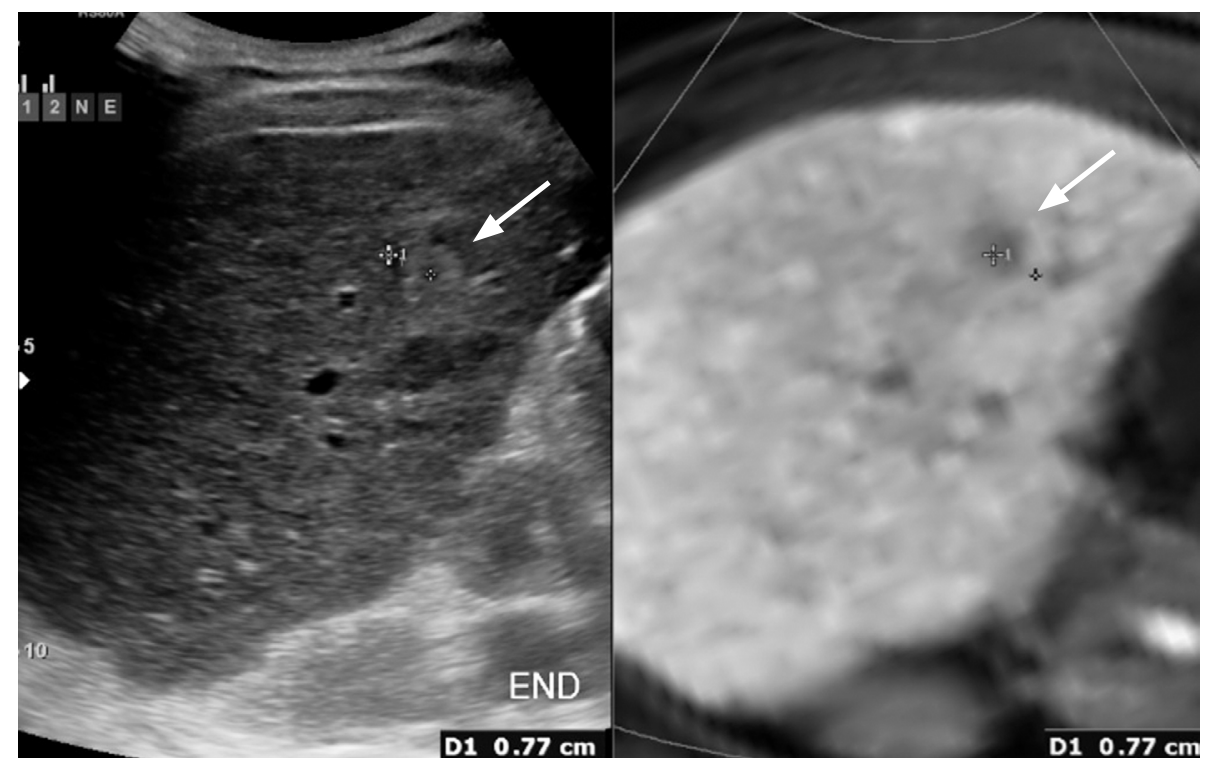

B
Fig. 2. The method of measuring the registration error.

A. Ultrasonography and magnetic resonance imaging images were registered and an index tumor (arrows) was shown on both images. $B$. The distance between the center of the index tumor (arrows) on both images was measured. Patients were asked to hold their breath to minimize registration error during measurement. 
significant differences. Statistical analyses were performed with SPSS version 24.0 (IBM Corp., Armonk, NY, USA) and MedCalc version 17.7 (MedCalc Software).

\section{Results}

\section{Patients}

From February 2016 to March 2017, a total of 123 patients were consulted for RFA. Eighty-two patients who provided written informed consent were randomly assigned to either automatic (41 patients) or manual (41 patients) registration (Fig. 3). Two patients in the automatic group did not undergo RFA because of duplicated enrollment in another clinical trial in one patient and withdrawal of informed consent in the other. A patient in the manual group was not included due to being younger than 40 years old, which was not in accordance with the protocol. Two patients in the automatic group were lost to follow-up after RFA. One patient in the automatic group discontinued RFA because of a technical error (malfunction of the US system). One patient in the manual group was excluded due to an inability to undergo follow-up contrast-enhanced CT or
MRI after RFA. We also excluded one patient in the automatic group and four patients in the manual group because their index tumors were smaller than $1 \mathrm{~cm}$, making it very difficult and inaccurate to measure the registration error for these very small tumors. Moreover, HCCs smaller than $1 \mathrm{~cm}$ cannot be diagnosed by imaging studies alone, and this factor could also affect the clinical outcomes. Finally, 70 patients were included in this study and the characteristics of the two groups are shown in Table 1.

\section{Technical Parameters}

Table 2 presents a comparison of the performance of both image fusion techniques. The registration error, time required for registration, and number of point registrations did not show significant differences between the automatic and manual registration techniques (Fig. 4).

Table 3 summarizes the subgroup analyses grouped by operator. The registration error, time required for registration, and number of point registrations were not significantly different between the two fusion techniques for either the more-experienced or lessexperienced operator. However, the time required for registration

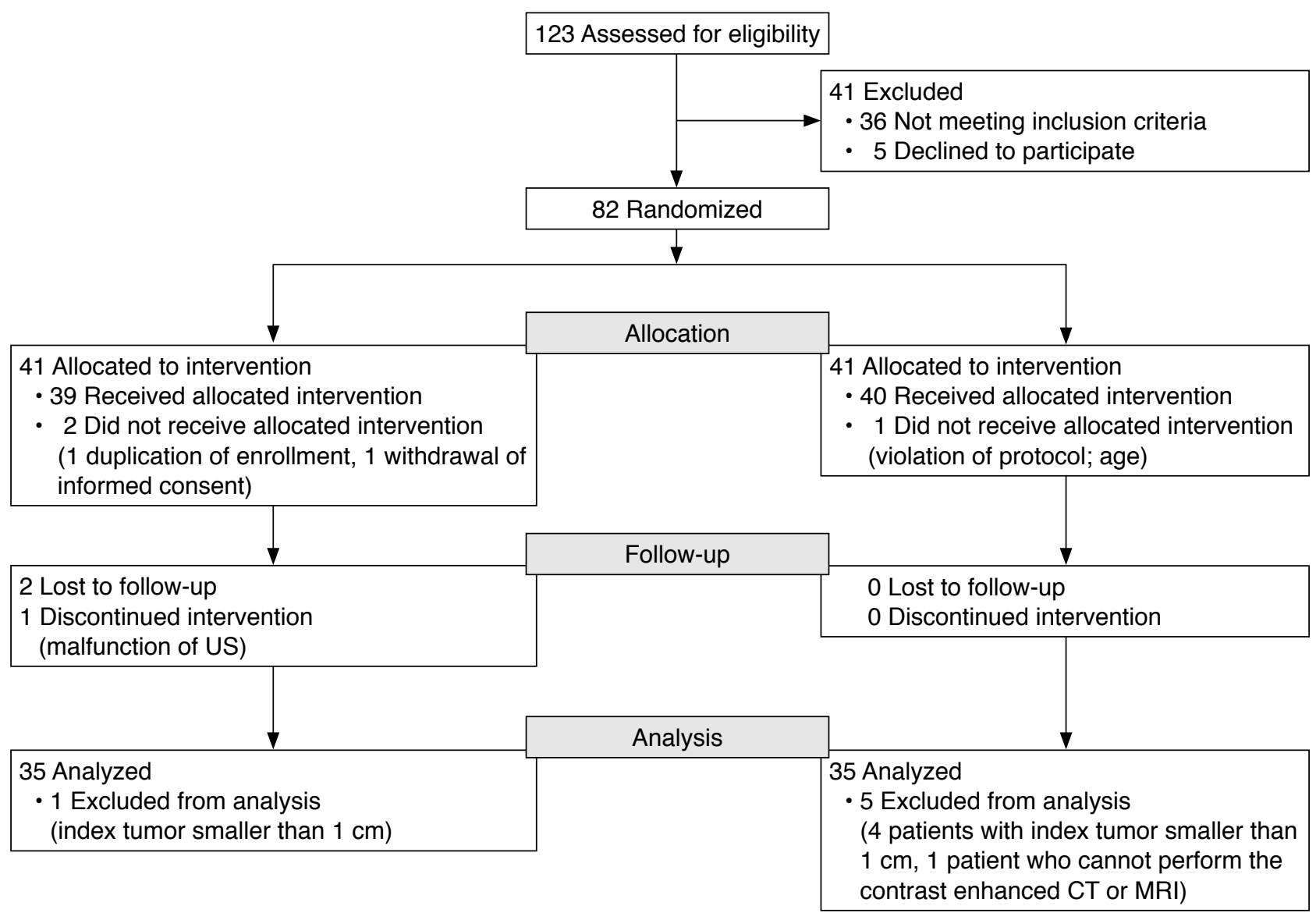

Fig. 3. A flow chart showing the enrollment of patients. US, ultrasonography; CT, computed tomography; MRI, magnetic resonance imaging. 
Table 1. Patient characteristics

\begin{tabular}{|c|c|c|c|}
\hline Characteristic & Automatic $(n=35)$ & Manual $(n=35)$ & P-value \\
\hline Age (year) & $67.3 \pm 11.2$ & $67.0 \pm 10.8$ & $0.905^{a)}$ \\
\hline Sex (male/female) & $24(68.6) / 11(31.4)$ & $23(65.7) / 12(34.3)$ & $>0.99^{\mathrm{b})}$ \\
\hline \multicolumn{4}{|l|}{ Tumor } \\
\hline $\begin{array}{l}\text { Tumor type } \\
\text { (HCC/CRLM) }\end{array}$ & $35(100) / 0(0)$ & $34(97.1) / 1(2.9)$ & \\
\hline Tumor number & $1.14 \pm 0.43$ & $1.11 \pm 0.32$ & $0.754^{a)}$ \\
\hline Index tumor size $(\mathrm{cm})$ & $1.63 \pm 0.59$ & $1.73 \pm 0.64$ & $0.449^{a)}$ \\
\hline $\begin{array}{l}\text { Index tumor location } \\
\text { (right/left) }\end{array}$ & $33(82.5) / 7(17.5)$ & $31(79.5) / 8(20.5)$ & $0.781^{b)}$ \\
\hline Liver cirrhosis & $28(80.0)$ & $27(77.1)$ & $>0.99^{b)}$ \\
\hline $\begin{array}{l}\text { Sonic window } \\
\text { (good/poor) }\end{array}$ & $34(97.1) / 1(2.9)$ & $32(91.4) / 3(8.6)$ & $0.614^{\mathrm{b})}$ \\
\hline Visibility of index tumor & & & $0.607^{c)}$ \\
\hline Very good & $12(34.3)$ & $10(28.6)$ & \\
\hline Good & $16(45.7)$ & $13(37.1)$ & \\
\hline Moderate & $2(5.7)$ & $3(8.6)$ & \\
\hline Poor & $5(14.3)$ & $9(25.7)$ & \\
\hline $\begin{array}{l}\text { Operators } \\
\text { (more-/less-experienced) }\end{array}$ & $27(77.1) / 8(22.9)$ & $21(60.0) / 14(40.0)$ & $0.197^{b)}$ \\
\hline Fused images (MRI/CT) & $34(97.1) / 1(2.9)$ & $32(91.4) / 3(8.6)$ & $0.614^{\text {b) }}$ \\
\hline
\end{tabular}

Values are presented as mean \pm standard deviation or number (\%).

HCC, hepatocellular carcinoma; CRLM, colorectal liver metastasis; MRI, magnetic resonance imaging; $\mathrm{CT}$, computed tomography.

${ }^{\text {a) }} \mathrm{P}$-values were calculated using the Student t-test. ${ }^{b}$ The Fisher exact test. ${ }^{\mathrm{c}}$ The chisquare test.

Table 2. Comparison of performance between manual and automatic registration in all patients

\begin{tabular}{lccc}
\hline & $\begin{array}{c}\text { Automatic } \\
(\mathrm{n}=35)\end{array}$ & $\begin{array}{c}\text { Manual } \\
(\mathrm{n}=35)\end{array}$ & P-value \\
\hline Registration error $(\mathrm{mm})$ & $5.7 \pm 4.3$ & $6.3 \pm 5.0$ & 0.604 \\
Time required for registration $(\mathrm{s})$ & $147.8 \pm 78.2$ & $150.3 \pm 89.7$ & 0.900 \\
No. of point registrations & $3.26 \pm 1.20$ & $3.20 \pm 1.13$ & 0.838 \\
\hline
\end{tabular}

Values are presented as mean \pm standard deviation.

P-values were calculated using the Student t-test.

was significantly shorter for the more-experienced operator than for the less-experienced operator in both methods. The registration error of the more-experienced operator using manual registration was significantly higher than that of the less-experienced operator. The registration errors using automatic registration and the number of point registrations in both fusion techniques were not significantly different between the more- and less-experienced operators.

\section{Clinical Outcomes}

There was only one case of technical failure and technical
Table 3. Subgroup analyses of performance by operator

\begin{tabular}{lccc}
\hline & Automatic & Manual & P-value \\
\hline Registration error (mm) & & & \\
More-experienced operator & $6.04 \pm 4.34$ & $7.35 \pm 4.68$ & 0.350 \\
Less-experienced operator & $4.63 \pm 4.14$ & $4.79 \pm 5.22$ & 0.868 \\
P-value (more- vs. less-experienced) & 0.537 & 0.022 & \\
Time required for registration (s) & & & \\
More-experienced operator & $125.6 \pm 68.2$ & $106.3 \pm 50.3$ & 0.366 \\
Less-experienced operator & $222.5 \pm 64.5$ & $216.4 \pm 96.6$ & 0.815 \\
P-value (more- vs. less-experienced) & 0.001 & 0.001 & \\
No. of point registrations & & & \\
More-experienced operator & $3.26 \pm 1.20$ & $2.86 \pm 0.91$ & 0.217 \\
Less-experienced operator & $3.25 \pm 1.28$ & $3.71 \pm 1.27$ & 0.238 \\
P-value (more- vs. less-experienced) & 0.954 & 0.097 & \\
\hline
\end{tabular}

Values are presented as mean \pm standard deviation.

P-values were calculated using the Mann-Whitney $\mathrm{U}$ test.

ineffectiveness in the automatic registration group, which was performed by the less-experienced operator. No case of technical failure or ineffectiveness was reported in the manual registration group. The technical success rate and technical effectiveness rate for automatic registration $(97.1 \%, 34$ of 35$)$ and manual registration $(100.0 \%, 35$ of 35$)$ were not significantly different.

The follow-up period for survival analysis was 1-34 months (median, 20.1 months). The mean LTP-free survival was 28.3 months (95\% confidence interval $[\mathrm{CI}], 26.0$ to 30.6 ) in the automatic registration group and 29.0 months $(95 \% \mathrm{Cl}, 27.1$ to 30.8$)$ in the manual registration group, which was not a significant difference $(P=0.565)$. The PFS was 21.2 months $(95 \% \mathrm{Cl}, 17.6$ to 24.7$)$ and 24.9 months $(95 \% \mathrm{Cl}, 22.4$ to 27.4$)$ in the automatic and the manual registration groups, respectively, and there was no significant difference ( $P=0.368$ ) (Fig. 5).

There were no immediate major complications in either group. Two patients in the automatic registration group had minor complications (self-limiting bleeding in one patient and diaphragm thickening in one patient) and one patient in the manual registration group had a minor complication (gallbladder wall thickening that did not need treatment).

\section{Discussion}

This prospective randomized study showed that there were no significant differences in fusion-related parameters or the technical success/effectiveness rate between automatic and manual registration of US-MR/CT fusion techniques for RFA of hepatic tumors. In addition, automatic imaging fusion showed similar 


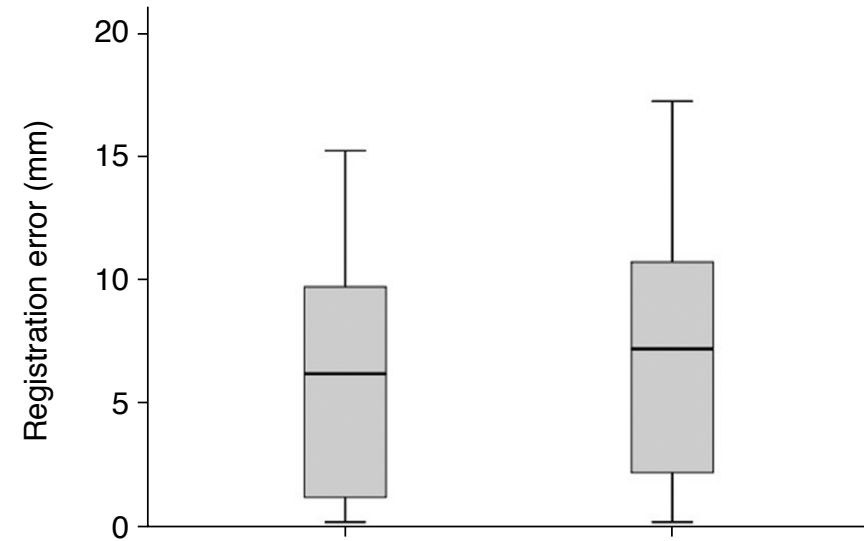

Automatic registration Manual registration

A

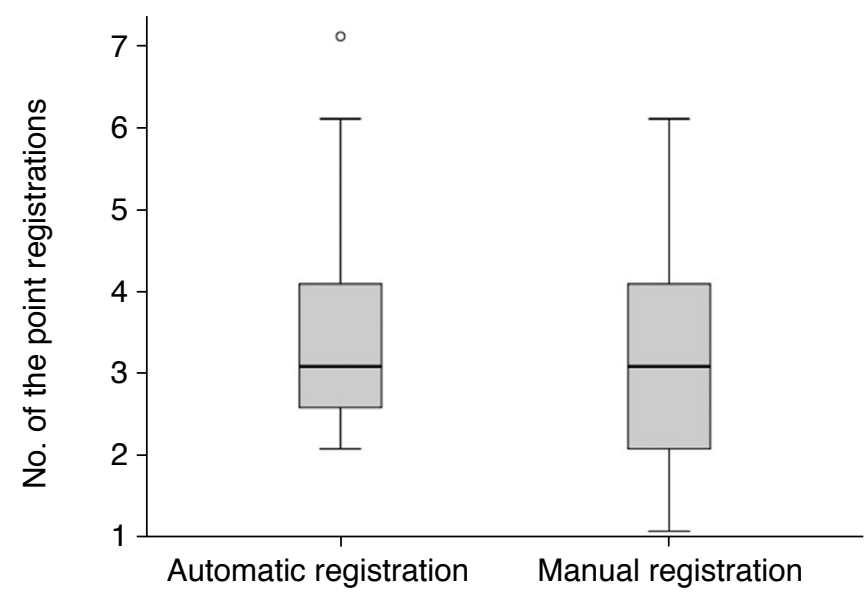

C

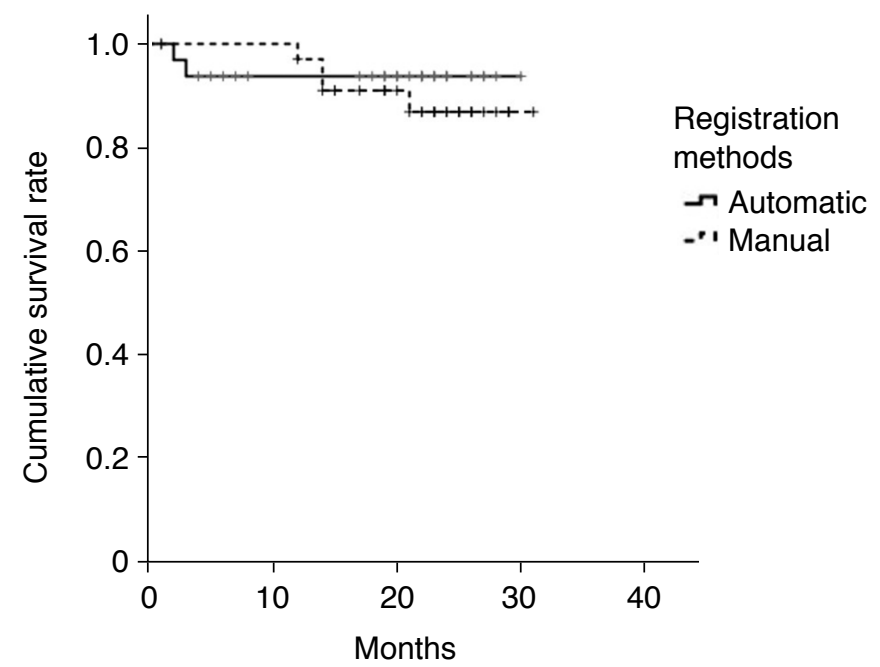

A

Fig. 5. Survival curves of local tumor progression-free survival and progression-free survival.

Survival curves by the Kaplan-Meier method show no significant difference in local tumor progression-free survival (A) and progression-free survival (B) between the automatic and manual registration groups.

Fig. 4. Box-and-whisker plots for technical parameters.

Registration error $(A)$, registration time $(B)$, and the number of the point registrations $(C)$ show no significant difference between the automatic and manual registration methods.

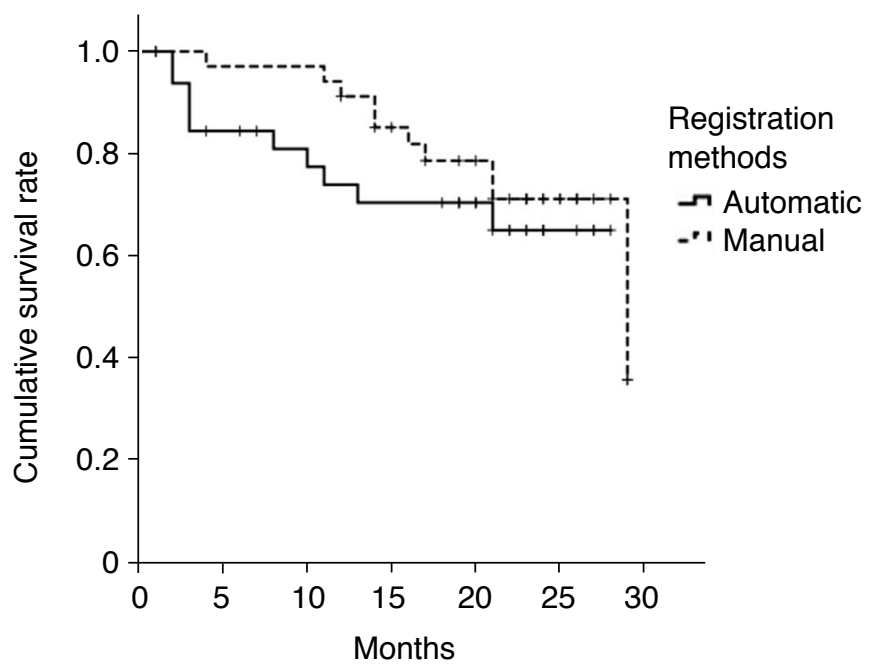

B

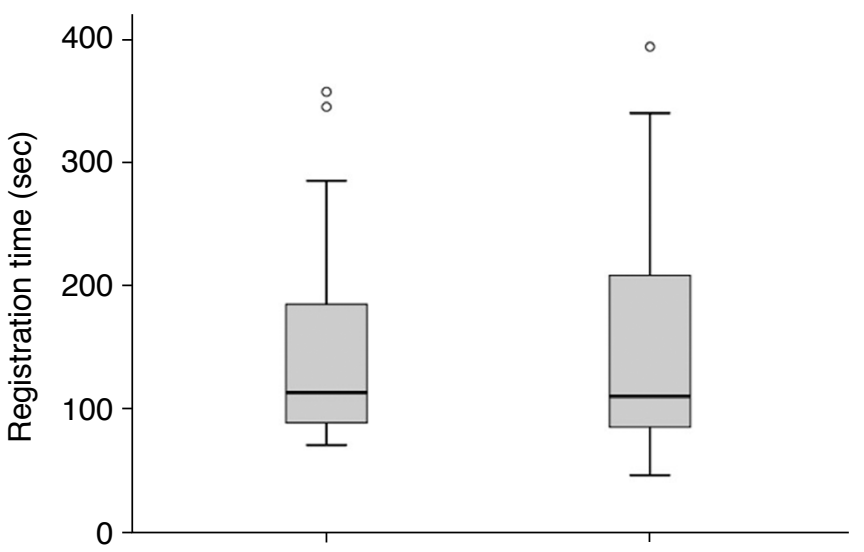

B 
clinical outcomes for RFA of hepatic malignancies. Considering its easy application, the automatic imaging fusion technique may be helpful for physicians performing interventional procedures targeting hepatic lesions under US guidance, especially for physicians with little experience.

In this study, there was no significant difference in the time required for registration and the number of point registrations between the two registration techniques. Our results are not consistent with the previous findings of Cha et al. [19], as their study showed a significantly shorter registration time for automatic registration than manual registration even though the number of point registrations was not significantly different between the two methods. Their study compared the time for plane and point registration during manual registration and the time for point registration during automatic registration. However, we calculated the required time for fusion starting from the end of data uploading to the completion of image fusion. Since plane registration is a common step in both techniques, we thought that including the time for automatic plane registration would be more reasonable for comparing the required time between the two methods.

The registration time was much shorter (28.5-36.5 seconds) in the previous study by Cha et al. [19] than in our study (106.3222.5 seconds). In the previous study, a single radiologist, an expert familiar with the US-CT/MRI fusion technique, performed both automatic and manual registration methods on each patient in a randomly determined order. Therefore, the radiologist's memory of the landmarks near the target tumor could have shortened the time required for completing the second fusion of US and MRI/CT. On the contrary, two operators with different experience levels performed image fusion in our study and they used one of the two fusion techniques for each patient. Therefore, a longer time for registration was inevitable in our study. We proved that there were no significant differences in the registration error, time required for registration, and number of point registrations between automatic and manual registration by both the more- and less-experienced operators. In most previous studies, image fusion was performed by an experienced operator $[19,20,29]$, meaning that their findings do not provide information on whether the performance of the two image fusion techniques by a less-experienced operator would be similar. Since our study included a relatively large number of patients and randomly allocated the patients to either the manual or automatic registration method, we believe that the difficulty of RFA was similar in both groups.

The less-experienced operator took significantly longer than the more-experienced operator for image fusion in both the automatic and manual registration techniques. However, the less-experienced operator did not use more point registrations. Unfamiliarity with image fusion may have caused difficulty in finding the same planes on US and CT/MRI and a longer fusion time. However, the registration error was higher in both registration methods by the more-experienced operator compared to the less-experienced operator, with a significant difference in manual registration. This may have also been due to differences in experience, as the moreexperienced operator used the fusion technique only to detect and check the location of the targeted tumor. Therefore, meticulous image fusion was not necessary. However, the less-experienced operator had less confidence in her/his RFA skills; therefore, she/ he finished image fusion when she/he was convinced that the largest part of the tumor was included in the plane on the basis of meticulous image fusion.

The technical success rate and technical effectiveness rate were not different between the two registration techniques. Regarding long-term clinical outcomes with a median 20.1-month follow-up period, LTP-free survival and PFS also were not significantly different according to the registration method used. Previous studies of the automatic fusion technique focused on fusion-related parameters such as the registration time and registration error $[19,21]$. Our study confirmed that the US-CT/MRI registration method did not affect technical success rate and or LTP-free survival after RFA in the liver. Once image fusion was completed, the registration performance was similar between the two registration methods in our study. Therefore, it can be postulated that the treatment outcomes after RFA would also be similar between the two registration methods, as long as the tumor characteristics are not significantly different.

In our study, the majority of the tumors were clearly visible (very good or good) and imaging fusion may not have been necessary in these cases, especially in terms of cost-effectiveness. However, even in cases with well-demarcated tumors, fusion techniques may be useful to improve the confidence of operators and to ensure that the RFA zone sufficiently covers the tumor.

Our study has several limitations. First, we used two different US machines for the automatic and manual registration methods, respectively. The process of image fusion may be vendor-specific, especially for automatic registration. Cross-comparison of two registration methods using two US machines would be better to remove the possibility of vendor-specific characteristics in fusion techniques. However, this was impossible, as the automatic fusion technique was available in only one machine. Second, we did not objectively measure the registration error, as in a previous study [19]. We used different US machines for manual and automatic registration and the software could not be applied to the images from a different vendor's machine. However, the measurement method in the current study was consistently used with images from 
two different machines, making it a practical way to measure the registration error. Third, the standard deviations of some parameters were large. Therefore, statistical significance was not proven for some parameters and only a trend was observed. The considerable differences in the difficulty of image fusion among patients caused many parameters to have large standard deviations.

In conclusion, the technical parameters and clinical outcomes of automatic image fusion were not significantly different from those of manual image fusion for RFA of hepatic tumors.

ORCID: Moon Hyung Choi: https://orcid.org/0000-0001-5962-4772; Joon-Il Choi: https://orcid.org/0000-0003-0018-8712; Young Joon Lee: https://orcid.org/00000001-8309-0272

\section{Author Contributions}

Conceptualization: Choi Jl, Lee YJ. Data acquisition: Choi MH, Choi Jl. Data analysis or interpretation: Choi MH, Choi Jl, Lee YJ. Drafting of the manuscript: Choi MH, Choi Jl. Critical revision of the manuscript: Choi MH, Choi Jl. Approval of the final version of the manuscript: all authors.

\section{Conflict of Interest}

Activities related to the present article: disclosed no relevant relationship (M.H.C., J.-I.C., Y.J.L.). Activities not related to the present article: author previously received grants from Guerbet Korea, author previously received honoraria from Siemens Healthineers (M.H.C.); author previously received grants from Bayer Healthcare, Guerbet Korea, Bracco Korea, GE Healthcare, Starmed, author previously received honoraria from Bayer Healthcare, Samsung Medison, Samsung Eletronics, Guerbet Korea, Bracco Korea (J.-I.C.); author previously received grants from Inventive Health Korea, Starmed, author previously received honoraria from Starmed (Y.J.L.). Other relationships: nothing to declare (M.H.C., J.-I.C., Y.J.L.).

\section{Acknowledgments}

This research was supported by a grant of the Korea Health Technology R\&D Project through the Korea Health Industry Development Institute (KHIDI), funded by the Ministry of Health \& Welfare, Republic of Korea (grant number: HI15C0833).

\section{References}

1. Korean Liver Cancer Association (KLCA); National Cancer Center. 2018 Korean Liver Cancer Association-National Cancer Center Korea Practice guidelines for the management of hepatocellular carcinoma. Korean J Radiol 2019;20:1042-1113.

2. Marrero JA, Kulik LM, Sirlin CB, Zhu AX, Finn RS, Abecassis MM, et al. Diagnosis, staging, and management of hepatocellular carcinoma: 2018 practice guidance by the American Association for the Study of Liver Diseases. Hepatology 2018;68:723-750.

3. European Association for the Study of the Liver. EASL clinical practice guidelines: management of hepatocellular carcinoma. J Hepatol 2018;69:182-236.

4. Omata M, Cheng AL, Kokudo N, Kudo M, Lee JM, Jia J, et al. Asia-Pacific clinical practice guidelines on the management of hepatocellular carcinoma: a 2017 update. Hepatol Int 2017:11:317-370.

5. Rhim H, Lee MH, Kim YS, Choi D, Lee WJ, Lim HK. Planning sonography to assess the feasibility of percutaneous radiofrequency ablation of hepatocellular carcinomas. AJR Am J Roentgenol 2008;190:1324-1330.

6. Lencioni R, Cioni D, Crocetti L, Franchini C, Pina CD, Lera J, et al. Early-stage hepatocellular carcinoma in patients with cirrhosis: long-term results of percutaneous image-guided radiofrequency ablation. Radiology 2005;234:961-967.

7. Lee MW, Kim YJ, Park HS, Yu NC, Jung SI, Ko SY, et al. Targeted sonography for small hepatocellular carcinoma discovered by $\mathrm{CT}$ or MRI: factors affecting sonographic detection. AJR Am J Roentgenol 2010;194:W396-W400.

8. Kim JE, Kim YS, Rhim H, Lim HK, Lee MW, Choi D, et al. Outcomes of patients with hepatocellular carcinoma referred for percutaneous radiofrequency ablation at a tertiary center: analysis focused on the feasibility with the use of ultrasonography guidance. Eur J Radiol 2011;79:e80-e84.

9. Yoon SH, Lee KH, Kim SY, Kim YH, Kim JH, Lee SH, et al. Realtime contrast-enhanced ultrasound-guided biopsy of focal hepatic lesions not localised on B-mode ultrasound. Eur Radio 2010;20:2047-2056.

10. Kim PN, Choi D, Rhim H, Rha SE, Hong HP, Lee J, et al. Planning ultrasound for percutaneous radiofrequency ablation to treat small $(\leq 3 \mathrm{~cm})$ hepatocellular carcinomas detected on computed tomography or magnetic resonance imaging: a multicenter prospective study to assess factors affecting ultrasound visibility. J Vasc Interv Radiol 2012;23:627-634.

11. Lee MW, Lim HK, Kim YJ, Choi D, Kim YS, Lee WJ, et al. Percutaneous sonographically guided radio frequency ablation of hepatocellular carcinoma: causes of mistargeting and factors affecting the feasibility of a second ablation session. J Ultrasound Med 2011;30:607-615.

12. Mauri G, Cova L, De Beni S, lerace T, Tondolo T, Cerri A, et al. Realtime US-CT/MRI image fusion for guidance of thermal ablation of liver tumors undetectable with US: results in 295 cases. Cardiovasc Intervent Radiol 2015;38:143-151.

13. Lee MW. Fusion imaging of real-time ultrasonography with $\mathrm{CT}$ or MRI for hepatic intervention. Ultrasonography 2014;33:227-239.

14. Liu FY, Yu XL, Liang P, Cheng ZG, Han ZY, Dong BW, et al. 
Microwave ablation assisted by a real-time virtual navigation system for hepatocellular carcinoma undetectable by conventional ultrasonography. Eur J Radiol 2012;81:1455-1459.

15. Ewertsen C, Henriksen BM, Torp-Pedersen S, Bachmann Nielsen M. Characterization by biopsy or CEUS of liver lesions guided by image fusion between ultrasonography and CT, PET/CT or MRI. Ultraschall Med 2011;32:191-197.

16. Minami Y, Chung H, Kudo M, Kitai S, Takahashi S, Inoue T, et al. Radiofrequency ablation of hepatocellular carcinoma: value of virtual CT sonography with magnetic navigation. AJR Am J Roentgenol 2008;190:W335-W341.

17. Lee J, Lee MW, Choi D, Cha DI, Lee S, Kang TW, et al. Active contour configuration model for estimating the posterior ablative margin in image fusion of real-time ultrasound and 3D ultrasound or magnetic resonance images for radiofrequency ablation: an experimental study. Ultrasonography 2018;37:337-344.

18. Cha DI, Lee MW, Kim AY, Kang TW, Oh YT, Jeong JY, et al. Automatic image fusion of real-time ultrasound with computed tomography images: a prospective comparison between two auto-registration methods. Acta Radiol 2017:58:1349-1357.

19. Cha Dl, Lee MW, Song KD, Oh YT, Jeong JY, Chang JW, et al. A prospective comparison between auto-registration and manual registration of real-time ultrasound with MR images for percutaneous ablation or biopsy of hepatic lesions. Abdom Radiol (NY) 2017:42:1799-1808.

20. Kim AY, Lee MW, Cha DI, Lim HK, Oh YT, Jeong JY, et al. Automatic registration between real-time ultrasonography and pre-procedural magnetic resonance images: a prospective comparison between two registration methods by liver surface and vessel and by liver surface only. Ultrasound Med Biol 2016;42:1627-1636.

21. Lee MW, Park HJ, Kang TW, Ryu J, Bang WC, Lee B, et al. Image fusion of real-time ultrasonography with computed tomography: factors affecting the registration error and motion of focal hepatic lesions. Ultrasound Med Biol 2017;43:2024-2032.
22. Mauri G, De Beni S, Forzoni L, D'Onofrio S, Kolev V, Lagana MM, et al. Virtual navigator automatic registration technology in abdominal application. Conf Proc IEEE Eng Med Biol Soc 2014;2014:55705574.

23. Nam WH, Kang DG, Lee $D$, Lee JY, Ra JB. Automatic registration between 3D intra-operative ultrasound and pre-operative $\mathrm{CT}$ images of the liver based on robust edge matching. Phys Med Biol 2012;57:69-91.

24. Wein W, Brunke S, Khamene A, Callstrom MR, Navab N. Automatic CT-ultrasound registration for diagnostic imaging and imageguided intervention. Med Image Anal 2008;12:577-585.

25. Bruix J, Sherman M; American Association for the Study of Liver Diseases. Management of hepatocellular carcinoma: an update. Hepatology 2011;53:1020-1022.

26. European Association for the Study of the Liver; European Organisation for Research, Treatment of Cancer. EASL-EORTC clinical practice guidelines: management of hepatocellular carcinoma. J Hepatol 2012;56:908-943.

27. Korean Liver Cancer Study Group; National Cancer Center Korea. 2014 Korean Liver Cancer Study Group-National Cancer Center Korea practice guideline for the management of hepatocellular carcinoma. Korean J Radiol 2015;16:465-522.

28. Lee MW, Rhim H, Cha DI, Kim YJ, Choi D, Kim YS, et al. Percutaneous radiofrequency ablation of hepatocellular carcinoma: fusion imaging guidance for management of lesions with poor conspicuity at conventional sonography. AJR Am J Roentgenol 2012;198:1438-1444.

29. Cha DI, Lee MW, Kang TW, Oh YT, Jeong JY, Chang JW, et al. Comparison between $\mathrm{CT}$ and MR images as more favorable reference data sets for fusion imaging-guided radiofrequency ablation or biopsy of hepatic lesions: a prospective study with focus on patient's respiration. Cardiovasc Intervent Radiol 2017;40:15671575. 